Jpn. J. Human Genet. 29, 39-43, 1984

\title{
A CASE OF SPONDYLOEPIPHYSEAL DYSPLASIA TARDA ASSOCIATED WITH OSTEOSARCOMA
}

\author{
Toshio Matsumoto, Yoshio Ogrhara, and Toyoshi Tsuruta \\ Department of Orthopaedic Surgery, Mie University School of Medicine, \\ Tsu 514, Japan
}

\begin{abstract}
Summary A case of osteosarcoma arising in a 16-year-old boy with spondyloepiphyseal dysplasia tarda is described.

The etiological interrelationship between these two conditions is uncertain. To our best knowledge, their coexistence has not previously been reported.
\end{abstract}

\section{INTRODUCTION}

Spondyloepiphyseal dysplasia tarda is a rare hereditary osteochondrodysplasia which manifests itself in adolescence or adult life as short stature with multiple epiphyseal lesions and results in premature osteoarthritis of the major joints of the extremities. The purpose of this paper is to describe a case of osteosarcoma in a boy with spondyloepiphyseal dysplasia tarda.

\section{CASE REPORT}

A 16-year-old boy without history of trauma was admitted to our hospital on Jan. 9, 1980, on account of the pain in his right knee for seven months.

The patient was of short stature but there was no family history of genetic disorders. Consanguinity of the parents was not observed (Fig. 1). The boy had become aware of his short stature at about age eleven. There was no mental retardation.

Physical examination showed short stature (height: $141 \mathrm{~cm}$ ). Though the extremities were of normal length, and all joint motion was of normal range, the trunk was markedly shortened and the thorax barrel-shaped (Fig. 2). A hard immobile mass was palpable on the upper part of his right leg.

Roentgenograms revealed an osteoblastic lesion with periosteal reaction at the proximal metaphysis of the right tibia (Fig. 3). Skeletal survey showed the flattening of all the vertebral bodies with a hump-shaped partial bulging (Fig. 4), and dysplastic changes in his hip and shoulder joints (Fig. 5).

Received September 16, 1983; revised version received January 27, 1984 


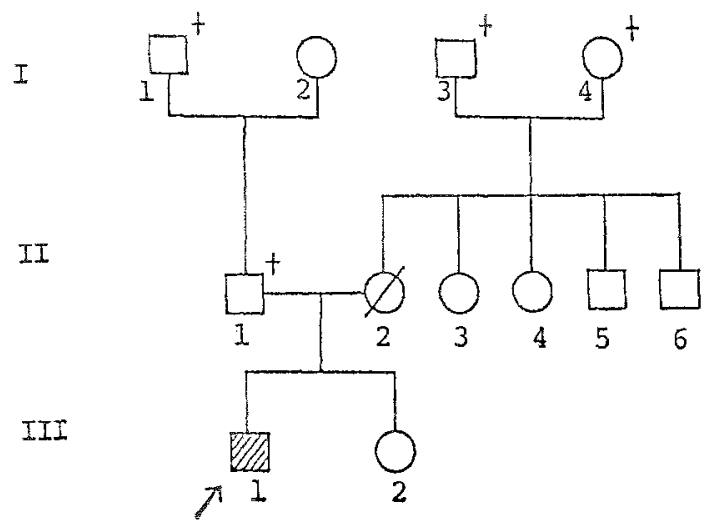

Fig. 1. Pedigree chart.

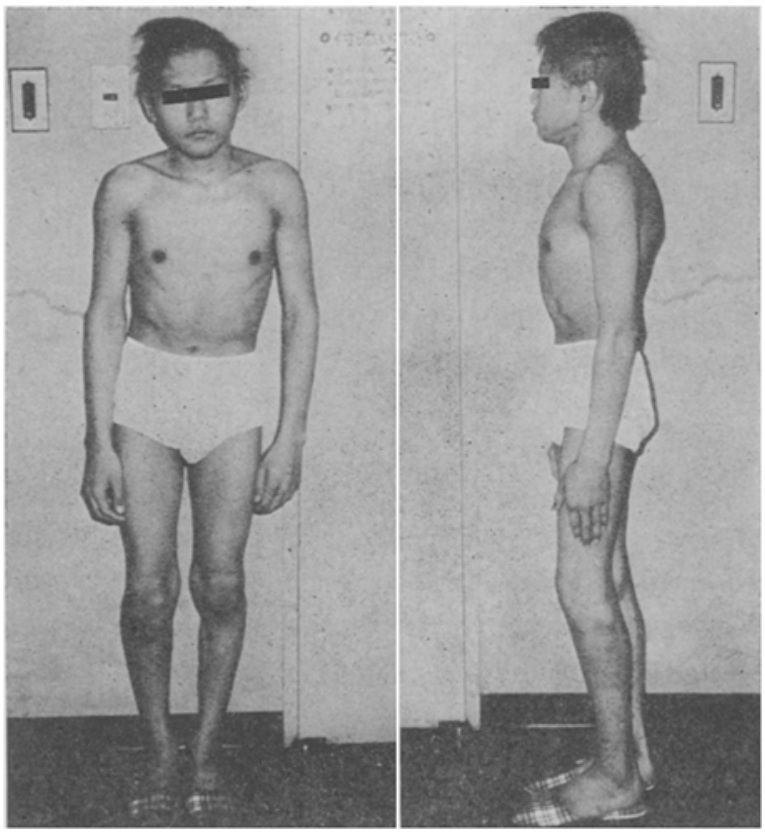

Fig. 2. Front and side view of the propositus. Short trunk type dwarfism is evident.

Routine laboratory examinations were within normal limits except for elevated alkaline phosphatase activity (252 international units per liter; normal 30 to 105 ).

The examination by an open biopsy of the right tibia lesion confirmed the diagnosis of osteosarcoma (Fig. 6). An above-knee amputation was performed on Feb. 7, 1980 . 


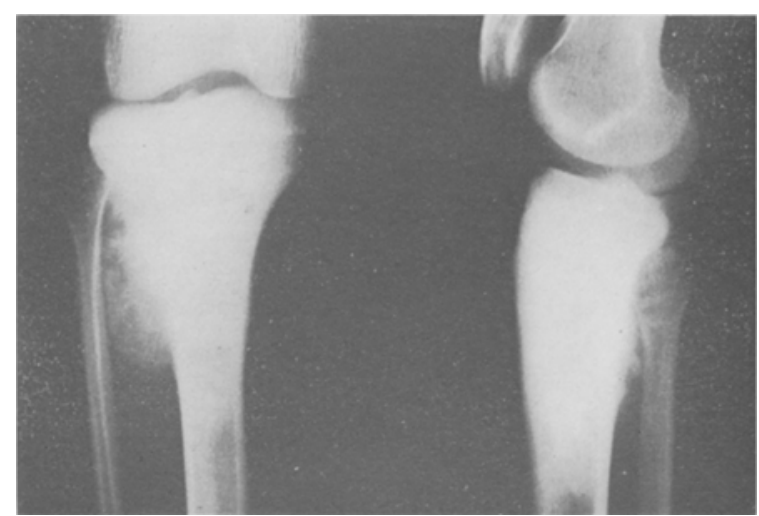

Fig. 3. Roentgenogram of right tibia showing an osteoblastic bone lesion with periosteal reaction.

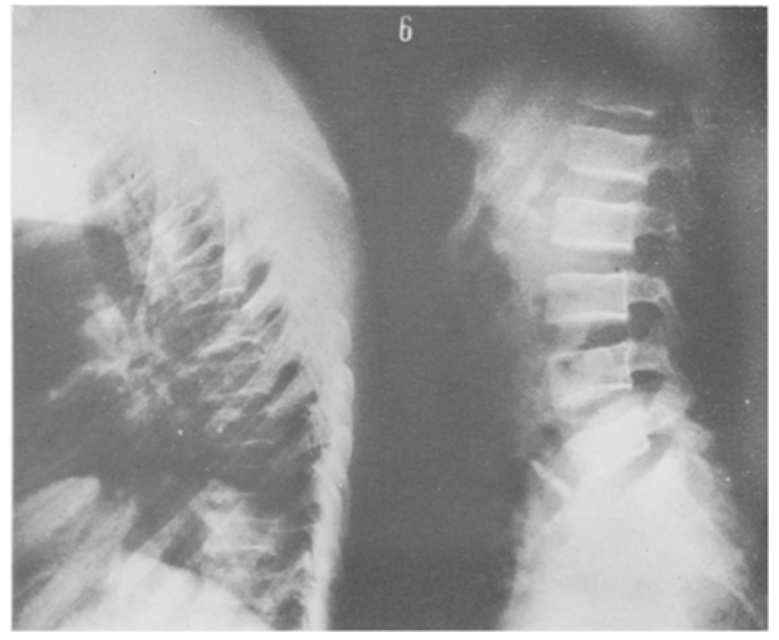

Fig. 4. Roentgenogram reveals flattening of all of the vertebral bodies.

Postoperatively, the patient was admitted to an adjuvant chemotherapy protocol (high-dose methotrexate plus adriamycin, cyclophosphamide and dactinomycin). These drugs were administered during a period of 60 weeks. At present, three years after the operation, the patient shows no evidence of recurrence or metastasis.

\section{COMMENT}

Spondyloepiphyseal dysplasia tarda, a rare hereditary disorder, was named by Maroteaux et al. in 1957. There have been many sporadic cases, and although a few 

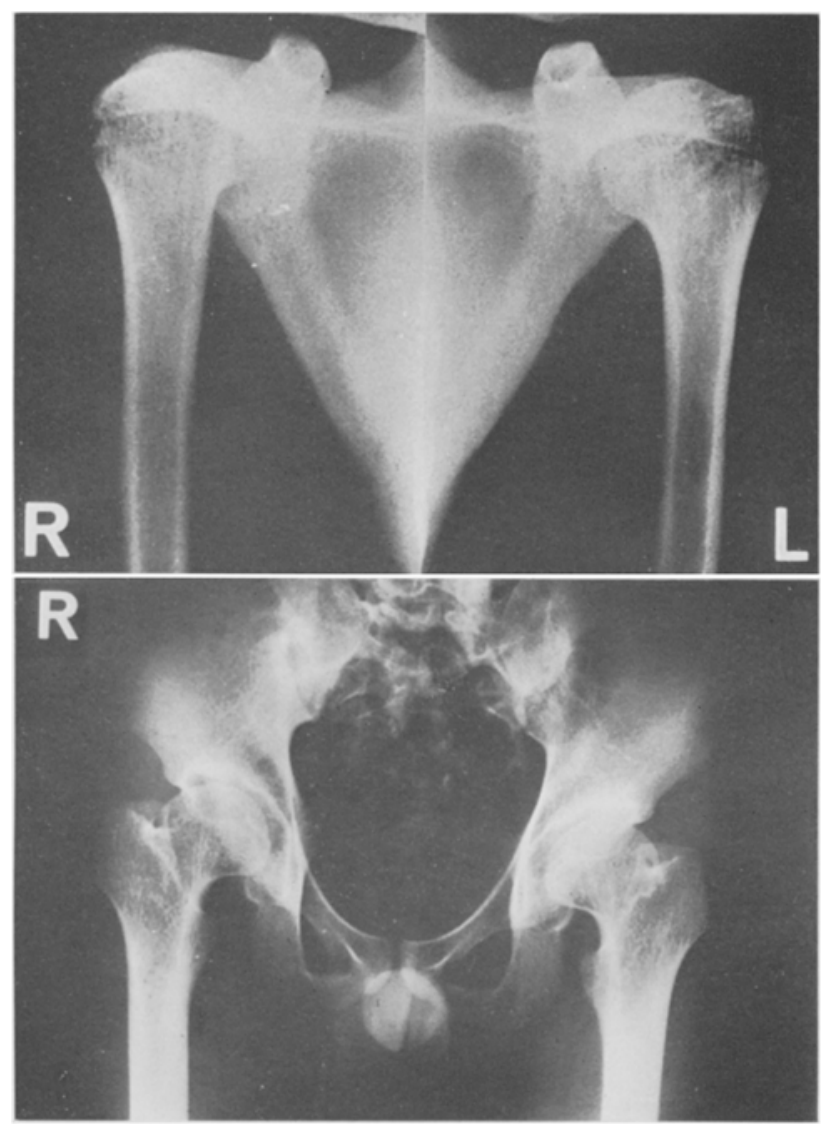

Fig. 5. Dysplastic changes in the hip and shoulder joints.

families with an autosomal dominant inheritance (Nakano et al., 1978) have been reported, spondyloepiphyseal dysplasia tarda is proved to be $\mathrm{X}$-linked recessive (McKusick, 1983). Bannerman et al. (1971) published diagnosis criteria for this disorder as follows:

1) X-linked recessive inheritance. 2) Short stature first evident in childhood between 5 and 14 years. 3) Shortness due to impaired growth of spine. 4) Radiologically, characteristic flattening of vertebrae with central humping. 5) Dysplastic changes of femoral heads and neck. 6) Minor changes in other bones.

The patient we present here appears to be a sporadic case, but the possibility of $\mathrm{X}$-linked inheritance cannot be entirely denied. Therefore, since the case is compatible with Bannerman's criteria, it seems reasonable to diagnose this case as spondyloepiphyseal dysplasia tarda.

Since spondyloepiphyseal dysplasia tarda is a rare congenital disease and osteosarcoma is also rare, the coexistence of the two conditions is extremely uncommon. 

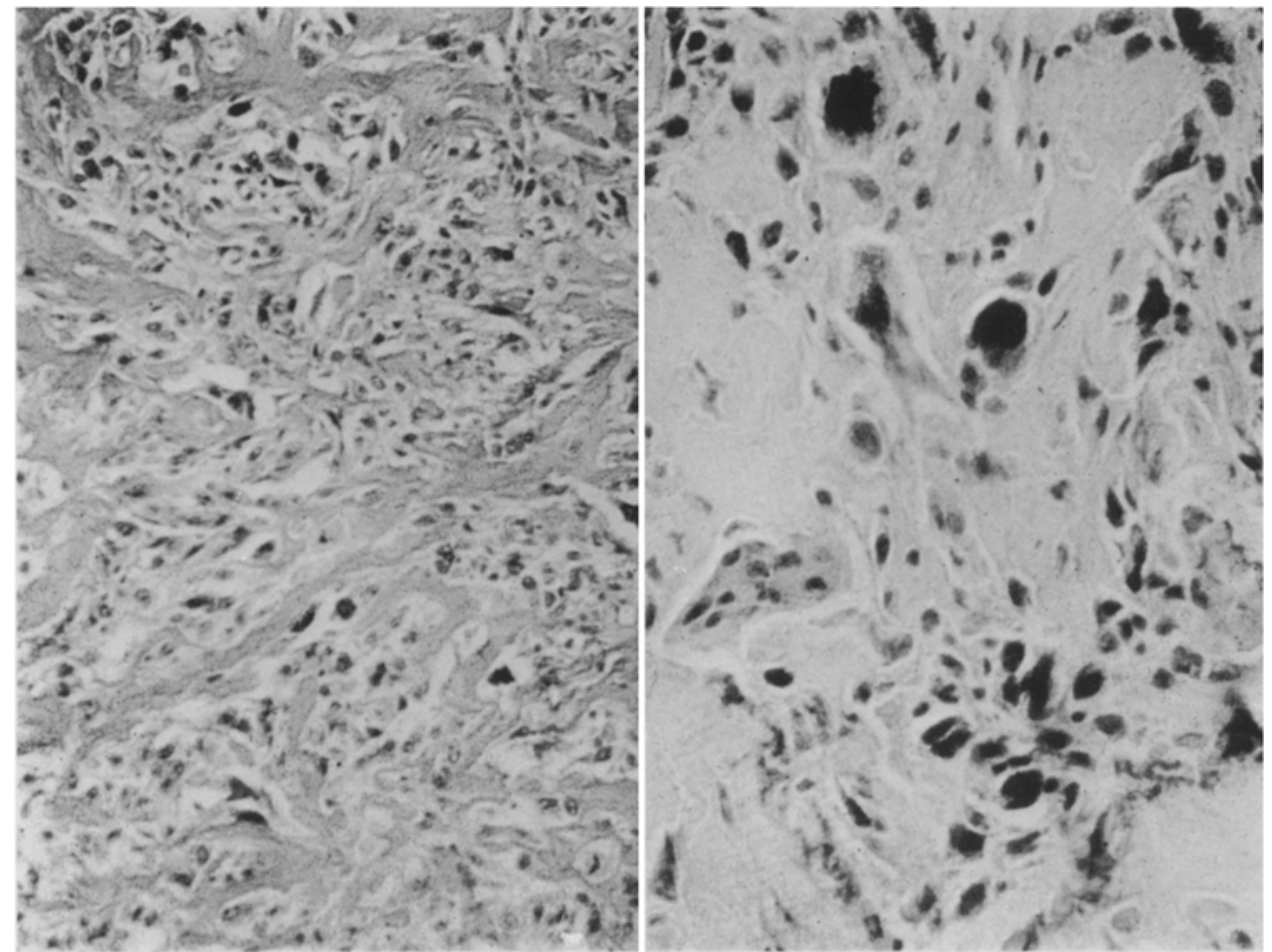

Fig. 6. Pathohistological specimen reveals typical osteosarcoma at the tibia showing a malignant spindle cell which is forming osteoid.

To our best knowledge, this is the first reported case of the coexistence of spondyloepiphyseal dysplasia tarda and osteosarcoma.

\section{REFERENCES}

Bannerman, R.M., Ingall, G.B., and Mohn, J.F. 1971. X-linked spondyloepiphyseal dysplasia tarda, clinical and linkage data. J. Med. Genet. 8 : 291-301.

Maroteaux, P., Lamy, M., and Bernard, J. 1957. La dysplasie spondylo-epiphysaire tardive: description clinique et radiologique. Presse Med. 65: 1205-1208.

Nakano, M., Kurata, K., Norimatsu, H., Takagi, K., Takahashi, H., Ito, S., and Kusano, K. 1978. 4 cases of spondyloepiphyseal dysplasia tarda. Tohoku Arch. Orthopaedic Surg. Traumatol. 21: 18-23 (in Japanese). 